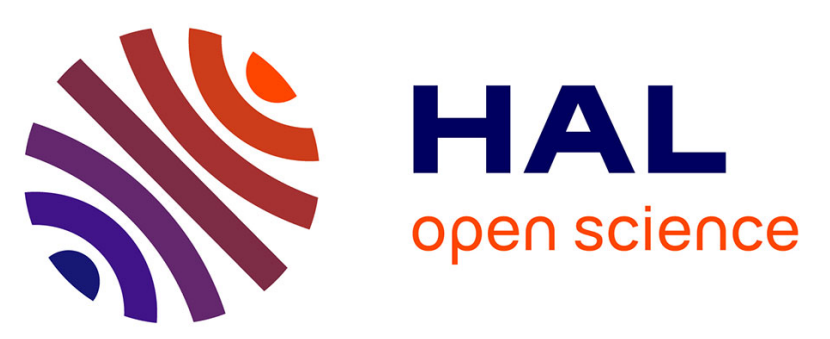

\title{
Inhibition of hepatic Carnitine Palmitoyl-Transferase I (CPT IA) by Valproyl-CoA as a possible mechanism of Valproate-induced steatosis
}

Cátia C.P. Aires, Lodewijk Ijlst, Femke Stet, Carina Prip-Buus, Isabel Tavares De Almeida, Marinus Duran, Ronald J.A. Wanders, Margarida F.B. Silva

\section{To cite this version:}

Cátia C.P. Aires, Lodewijk Ijlst, Femke Stet, Carina Prip-Buus, Isabel Tavares De Almeida, et al.. Inhibition of hepatic Carnitine Palmitoyl-Transferase I (CPT IA) by Valproyl-CoA as a possible mechanism of Valproate-induced steatosis. Biochemical Pharmacology, 2009, 79 (5), pp.792. 10.1016/j.bcp.2009.10.011 . hal-00547637

\section{HAL Id: hal-00547637 https://hal.science/hal-00547637}

Submitted on 17 Dec 2010

HAL is a multi-disciplinary open access archive for the deposit and dissemination of scientific research documents, whether they are published or not. The documents may come from teaching and research institutions in France or abroad, or from public or private research centers.
L'archive ouverte pluridisciplinaire HAL, est destinée au dépôt et à la diffusion de documents scientifiques de niveau recherche, publiés ou non, émanant des établissements d'enseignement et de recherche français ou étrangers, des laboratoires publics ou privés. 


\section{Accepted Manuscript}

Title: Inhibition of hepatic Carnitine Palmitoyl-Transferase I (CPT IA) by Valproyl-CoA as a possible mechanism of Valproate-induced steatosis

Authors: Cátia C.P. Aires, Lodewijk IJlst, Femke Stet, Carina Prip-Buus, Isabel Tavares de Almeida, Marinus Duran, Ronald

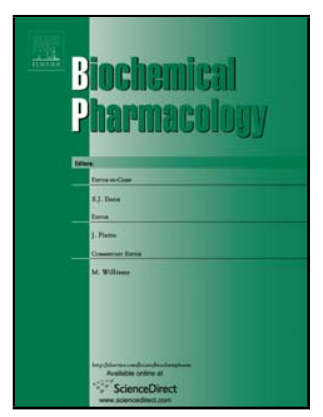

J.A. Wanders, Margarida F.B. Silva

PII:

S0006-2952(09)00905-8

DOI: doi:10.1016/j.bcp.2009.10.011

Reference: BCP 10357

To appear in: $\quad B C P$

Received date: $\quad$ 19-8-2009

Revised date: $\quad 14-10-2009$

Accepted date: $\quad$ 14-10-2009

Please cite this article as: Aires CCP, IJlst L, Stet F, Prip-Buus C, Almeida IT, Duran M, Wanders RJA, Silva MFB, Inhibition of hepatic Carnitine Palmitoyl-Transferase I (CPT IA) by Valproyl-CoA as a possible mechanism of Valproate-induced steatosis, Biochemical Pharmacology (2008), doi:10.1016/j.bcp.2009.10.011

This is a PDF file of an unedited manuscript that has been accepted for publication. As a service to our customers we are providing this early version of the manuscript. The manuscript will undergo copyediting, typesetting, and review of the resulting proof before it is published in its final form. Please note that during the production process errors may be discovered which could affect the content, and all legal disclaimers that apply to the journal pertain. 


\section{Inhibition of hepatic Carnitine Palmitoyl-Transferase I (CPT IA) by Valproyl-CoA as a} possible mechanism of Valproate-induced steatosis

Cátia C. P. Aires ${ }^{1,2}$, Lodewijk IJlst ${ }^{2}$, Femke Stet $^{2}$, Carina Prip-Buus ${ }^{3}$, Isabel Tavares de Almeida ${ }^{1}$, Marinus Duran ${ }^{2}$, Ronald J. A. Wanders ${ }^{2 \S}$, Margarida F. B. Silva ${ }^{1}{ }^{\S}$

${ }^{1}$ Research Institute for Medicines and Pharmaceutical Sciences - iMED.UL, Metabolism and Genetics group, Centro de Patogénese Molecular, Faculdade de Farmácia da Universidade de Lisboa, Av. Prof. Gama Pinto, 1649-003 Lisboa, Portugal;

${ }^{2}$ Laboratory Genetic Metabolic Diseases, Department of Clinical Chemistry and Pediatrics, Academic Medical Centre, University of Amsterdam, Meibergdreef 9, 1105 AZ Amsterdam, The Netherlands;

${ }^{3}$ Institut Cochin, Département d'Endocrinologie, Métabolisme et Cancer, Université Paris Descartes, CNRS Unité Mixte de Recherche 8104, and INSERM U567, 24 rue du Faubourg SaintJacques, 75014 Paris, France.

\section{${ }^{\S}$ These authors should be considered as equal last authors.}

Running title: Valproyl-CoA inhibits CPT IA activity

* Corresponding Author: Margarida F. B. Silva, iMED.UL, Centro de Patogénese Molecular, Faculdade de Farmácia da Universidade de Lisboa, Av. Prof. Gama Pinto, 1649-003 Lisboa, Portugal.

Tel./Fax: (+351) 2179464 91. E-mail: mbsilva@ @ff.ul.pt

Abbreviations: CPT I, carnitine palmitoyl-transferase I; VPA, valproic acid or 2- $n$-propylpentanoic acid; $\Delta^{4}$ VPA, $\Delta^{4}$-valproic acid or 2-n-propyl-4-pentenoic acid; CoA, coenzyme A; VP-CoA, valproyl-CoA; $\Delta^{4}$-VPCoA, $\Delta^{4}$-valproyl-CoA; AC, acylcarnitine; LCFA, long-chain fatty acids; FAO, fatty acid $\beta$-oxidation; LCFAO, long-chain fatty acid oxidation; BCA, bicinchoninic acid; ACN, acetonitrile; HSA, human serum albumin; C16-CoA, palmitoyl-CoA; Mal-CoA, malonyl-CoA; ESI-MS/MS, electrospray ionization tandem mass spectrometry. 


\begin{abstract}
Background/Aims: Carnitine palmitoyl-transferase I (CPT I) catalyses the synthesis of longchain(LC)-acylcarnitines from LC-acyl-CoA esters. It is the rate-limiting enzyme of mitochondrial fatty acid $\beta$-oxidation (FAO) pathway and its activity is regulated by malonyl-CoA. The antiepileptic drug valproic acid (VPA) is a branched chain fatty acid that is activated to the respective CoA ester in the intra- and extra-mitochondrial compartments. This drug has been associated with a clear inhibition of mitochondrial FAO, which motivated our study on its potential effect on hepatic CPT I.

Methods: To investigate the effect of valproyl-CoA (VP-CoA) on CPT I, we performed in vitro studies using control human fibroblasts and rat CPT IA expressed in Saccharomyces cerevisiae. In addition to the wild-type enzyme, two mutant rCPT IA's were studied, one of which showing increased sensitivity towards malonyl-CoA (S24A/Q30A), whereas the other one is insensitive to malonyl-CoA (E3A).

Results: We demonstrate that VP-CoA inhibits the CPT I activity in control fibroblasts. Similar results were obtained using rCPT IA WT and S24A/Q30A. Importantly, VP-CoA also inhibited the activity of the rCPT IA E3A. We show that VP-CoA inhibits CPT IA competitively with respect to palmitoyl-CoA, and non-competitively to carnitine. Evidence is provided that VP-CoA interferes at the catalytic domain of CPT IA affecting the sensitivity for malonyl-CoA.

Conclusions: The interference of VP-CoA with CPT IA, a pivotal enzyme in mitochondrial fatty acid $\beta$-oxidation, may be a crucial mechanism in the drug-induced hepatotoxicity and the weight gain frequently observed in patients under VPA therapy.
\end{abstract}

\title{
Word count of the abstract: 245 words
}

Key words: Carnitine Palmitoyl-transferase I; Valproic acid; Malonyl-CoA; Fatty acid oxidation; Drug induced hepatic steatosis 


\section{Introduction}

Carnitine palmitoyl-transferase I (CPT I, EC 2.3.1.21) is a mitochondrial outer membrane protein that is part of the carnitine shuttle, and catalyses the conversion of cytosolic long-chain (LC) acylCoA esters to the respective LC-acylcarnitine (AC) esters, allowing the transfer of LC-fatty acylCoA's into the mitochondrial matrix for $\beta$-oxidation $[1,2]$. The activity of this enzyme is inhibited by malonyl-CoA $[3,4]$, which is the carboxylation product of acetyl-CoA mediated by acetyl-CoA carboxylase (ACC) [5], and is also an intermediate in the de novo synthesis of LC-fatty acids (LCFA) [5-7]. It has been shown that malonyl-CoA regulates mitochondrial fatty acid oxidation (FAO) in a variety of tissues, including the liver, muscle, the pancreatic $\beta$-cell, endothelium, adipocytes and the central nervous system [6]. Consequently, a rise in malonyl-CoA levels (during a fed state) will decrease the mitochondrial fatty acid uptake and oxidation, whereas a decrease in malonyl-CoA (during starvation) promotes LCFA oxidation, since CPT I becomes uninhibited $[5,8]$.

Valproic acid (VPA) is currently the most widely used anticonvulsive drug worldwide, prescribed for the control of epileptic episodes. This C8-branched chain fatty acid is mainly metabolized in mitochondria by FAO, and via a minor pathway in the endoplasmatic reticulum, yielding $\Delta^{4}$ valproic acid. Both free acids can be activated to valproyl-CoA (VP-CoA) and $\Delta^{4}$-valproyl-CoA $\left(\Delta^{4}\right.$-VP-CoA $)$, respectively, not only in the mitochondrial matrix but also in the extramitochondrial compartment [9]. In mitochondria, VP-CoA can be fully metabolized by $\beta$-oxidation $[10,11]$ to acetyl- and propionyl-CoA, but its metabolic fate in the extra-mitochondrial space is still unknown. Consequently, these CoA esters may potentially affect numerous cellular functions besides mitochondrial FAO, triggering important consequences for the imbalance of the energetic state of the cell and for the metabolic fate of the drug.

Three different CPT I isoforms have been described in mammalian tissues: the liver (L-CPT I or CPT IA), muscle (M-CPT I or CPT IB) and brain (CPT IC) isoforms, which are encoded by different genes [12-14]. The kinetic characteristics of CPT IA and CPT IB differ in several 
important aspects. CPT IB is more sensitive to malonyl-CoA [2,15-17] whereas CPT IA has higher affinity for L-carnitine, one of the substrates $[16,18,19]$. The requirement for carnitine and the sensitivity to malonyl-CoA appear to be inversely related [20].

CPT IA is unique in responding to different physiological states (e.g. starvation and insulin deficiency) by changing its sensitivity to malonyl-CoA several fold. This occurs as a response to changes in the lipid composition of the membrane where it is localized, and with which it interacts through its two transmembrane (TM) domains. The polytopic membrane topology of the protein results in both a short regulatory N-terminal segment and a large catalytic C-terminal segment, both exposed to the cytosolic side of the outer mitochondrial membrane $[1,2,15,16,18]$. The $\mathrm{N}$ terminal domain, which contains both TM1 and TM2 domains, was shown to be responsible for mitochondrial import and for maintenance of a folded enzymatically active and malonyl-CoAsensitive conformation [19]. Moreover, the nature of the cytosolic N-C (N- and C-terminal domain) interactions determine the degree of malonyl-CoA sensitivity of the liver isoform [2,21]. Faye et al demonstrated that mutations in the regulatory regions of the $\mathrm{N}$-terminal domain affect the ability of this segment to interact physically with the C-terminal domain, either by increasing [S24A/Q30A] or lowering [E3A] the sensitivity of CPT I for malonyl-CoA [2]. CPT IA adopts different conformational states that differ in their degree of proximity between the cytosolic Nterminal and the C-terminal domains (intramolecular N/C interactions), and this determines its degree of malonyl-CoA sensitivity depending on the physiological state $[1,2,15,18,22-24]$. Recently it was shown that the sequence spanning the intermembrane loop-TM2 boundary determines the disposition of this TM in the membrane so as to alter the conformation of the Cterminal catalytic domain, and thus malonyl-CoA sensitivity $[1,23,24]$.

Over the last decades it has become clear that the role of the malonyl-CoA-CPT I interaction is crucial both in hepatic and nonlipogenic tissues, such as heart, skeletal muscle, pancreatic $\beta$-cell. It regulates glucose and fatty acid metabolism in response to different physiological and hormonal states. In several clinical studies involving patients subjected to VPA treatment, a significant weight gain is often described [25-27] as an unwanted side effect. Valproic acid-related weight gain was originally reported to be associated with hyperinsulinemia, probably explained by the fact 
that VPA might inhibit the metabolism of insulin in the liver. However, it seems that VPA-induced hyperinsulinemia is independent of the drug-related weight gain, and may actually precede weight gain [26]. The mechanisms responsible for this side effect associated with VPA therapy are still not clear and require clarification.

The present studies were designed to test the hypothesis that VP-CoA and $\Delta^{4}$-VP-CoA, formed in the cytosol, may interact with the carnitine shuttle at the level of CPT I activity, since the membrane topography of this enzyme dictates that both the active and regulatory (malonyl-CoAbinding) sites of CPT I are exposed to the cytosolic face of the outer membrane $[1,15,16,18,23,24]$. Our results unequivocally demonstrate that both CoA esters derived from VPA interfere with CPT IA activity, affecting its sensitivity to malonyl-CoA and its intrinsic regulation. As a consequence, the rate of LCFA oxidation and the regulation of mitochondrial FAO is affected, as previously reported by our group $[11,28]$. This may partially explain the reported weight gain and fatty liver associated with VPA treatment. 


\section{Materials and Methods}

\subsection{Chemicals}

VPA, HSA, L-carnitine, malonyl-CoA, bicinchoninic acid (BCA), digitonin and other standard biochemicals were obtained from Sigma-Aldrich. Complete mini protease inhibitor cocktail tablets were from Roche. The cell culture medium F-10 (Ham) Nutrient Mixture (25 mM HEPES + L-Glutamine) and the Trypsin-EDTA solution were acquired from Gibco. Potassium cyanide, butanol and acetylchloride were from Merck. Acetonitrile (ACN) gradient grade was obtained from Biosolve. The $\left[{ }^{2} \mathrm{H}\right]_{3}-\mathrm{C} 3,\left[{ }^{2} \mathrm{H}\right]_{3}-\mathrm{C} 8$ and $\left[{ }^{2} \mathrm{H}\right]_{3}-\mathrm{C} 16$-acylcarnitines internal standards were obtained from Dr. H.J. ten Brink (VUMC - Vrije University Medical Center, Amsterdam, The

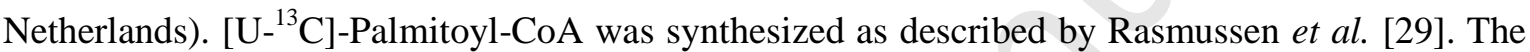
rCPT IA plasmids (wild type, [S24A/Q30A] and [E3A]) were obtained as described in [2].

\subsection{Synthesis of valproyl-CoA, $\Delta^{4}$-valproic acid and $\Delta^{4}$-valproyl-CoA}

Valproyl-CoA and $\Delta^{4}$-valproyl-CoA were synthesized according to published procedures [30] from VPA and $\Delta^{4}$-VPA, respectively. $\Delta^{4}$-Valproic acid was obtained by chemical synthesis following a reported procedure [31].

\subsection{Cells preparation}

Human control fibroblasts were cultured in F-10 (Ham) medium as described in [32]. The cells were harvested by trypsinization and washed twice with PBS. The protein content was measured by the bicinchoninic acid (BCA) method, using human serum albumin (HSA) as standard [33].

\section{4. rCPT IA plasmid expression in S. cerevisiae}

Three rat CPT IA forms were studied: (i) wild type (WT) rCPT IA; (ii) mutant protein rCPT IA (S24A/Q30A) with increased sensitivity to malonyl-CoA; and (iii) mutant protein rCPT IA (E3A) which is insensitive to malonyl-CoA (2). The WT and mutant rCPT IA's were expressed in the 
Saccharomyces cerevisiae strain BY4742 MAT $\alpha$ his $3 \Delta 1$ leu2 $\Delta 0$ lys $2 \Delta 0$ ura3 $\Delta 0 \Delta C A T 2$ (ATCC number $4016728^{\mathrm{TM}}$ from Invitrogen). The cells were grown in glucose medium (20 g/L glucose, $6.7 \mathrm{~g} / \mathrm{L}$ yeast nitrogen base (without amino acids), and amino acids ( $0.3 \mathrm{~g} / \mathrm{L} \mathrm{Leu,} 0.2 \mathrm{~g} / \mathrm{L}$ Try, 0.2 $\mathrm{g} / \mathrm{L}$ His, $0.3 \mathrm{~g} / \mathrm{L} \mathrm{Lys}$ ) for $24 \mathrm{~h}$ at $28^{\circ} \mathrm{C}$ in a gyro shaker, harvested by centrifugation and transfered to galactose medium (20 g/L galactose, $6.7 \mathrm{~g} / \mathrm{L}$ yeast nitrogen base, amino acids and $1 \mathrm{~g} / \mathrm{L}$ yeast extract). The cells from overnight cultures were harvested again by centrifugation and protoplasts were prepared using the lytic enzyme zymolyase, as described elsewhere [34]. The resulting protoplasts were centrifuged $(700 \times \mathrm{g}, 5 \mathrm{~min})$ and the obtained pellets were stored at $-80^{\circ} \mathrm{C}$ until usage for enzyme activity measurements. Protein concentration in the homogenates was determined using BCA solution and HSA as standard.

\subsection{CPT I activity measurement: in vitro assays}

The CPT I activity in human fibroblasts was measured in vitro after incubating the cells (final protein concentration of $0.5 \mathrm{mg} / \mathrm{mL}$, unless indicated otherwise) with $25 \mu \mathrm{M}\left[\mathrm{U}_{-}{ }^{13} \mathrm{C}\right]$-palmitoylCoA (C16-CoA), $0.5 \mathrm{mM} \mathrm{L-carnitine,} 5 \mathrm{mM} \mathrm{KCN}$ and $40 \mu \mathrm{g} / \mathrm{mL}$ digitonin, $37^{\circ} \mathrm{C}$, for 10 minutes. The used concentration of digitonin was formerly optimized in order to ensure the selective permeabilitzation of the plasma membrane, which allows VP-CoA to enter the cell without affecting the mitochondrial permeability and CPT I activity [35]. The activity of CPT I was evaluated in the presence of its specific inhibitor malonyl-CoA $(0-1 \mathrm{mM})$. The function of CPT I was further characterized in the presence of valproyl-CoA $(10-100 \mu \mathrm{M})$ or $\Delta^{4}$-valproyl-CoA $(100$ and $250 \mu \mathrm{M})$ after incubation at $37^{\circ} \mathrm{C}$ for 10 minutes.

The cells extracts of the three yeast strains expressing rCPT IA proteins $(10 \mu \mathrm{g} / \mathrm{mL}$ final concentration suspended in PBS + protease inhibitors) were sonicated $3 \times 10 \mathrm{sec}(8 \mathrm{~W})$ and used for CPT I activity measurements concerning the malonyl-CoA inhibition $(0-0.5 \mathrm{mM})$ and their affinities for the substrates, $\left[\mathrm{U}_{-}{ }^{13} \mathrm{C}\right]-\mathrm{C} 16-\mathrm{CoA}(0-50 \mu \mathrm{M})$ and L-carnitine $(0-750 \mu \mathrm{M})$, in parallel with the interference of valproyl-CoA $(0-100 \mu \mathrm{M})$.

The CPT I activity was measured as the synthesis rate of $\left[\mathrm{U}-{ }^{13} \mathrm{C}\right] \mathrm{C} 16$-carnitine, analysed and quantified by ESI-MS/MS [35]. 
A mixture of acylcarnitines (AC) internal standards (50 pmoles $\left[{ }^{2} \mathrm{H}_{3}\right]$-propionyl-carnitine (C3-AC), 50 pmoles $\left[{ }^{2} \mathrm{H}_{3}\right]$-octanoyl-carnitine (C8-AC) and 25 pmoles $\left[{ }^{2} \mathrm{H}_{3}\right]$-palmitoyl-carnitine (C16-AC)) was added to each sample. Each sample derived from the different incubations was analyzed in duplicate, and the assays for malonyl-CoA and palmitoyl-CoA with yeast expressed rCPT IA were performed twice. The mean values of the two independent experiments were used for calculation. The differential quantification of fatty acylcarnitines was performed using ESI-MS/MS as described previously [35]. The peak height ratio of the formed $\left[\mathrm{U}-{ }^{13} \mathrm{C}\right]-\mathrm{C} 16$-carnitine to the peak height of the internal standard $\left[{ }^{2} \mathrm{H}_{3}\right]-\mathrm{C} 16$-carnitine was determined using MassLynx NT software version 4.0 (Waters - Micromass, Manchester, UK). This ratio was used to calculate the CPT IA activity.

\subsection{Data analysis}

The characterization of the CPT I activity with different inhibitors was performed by plotting the measured reaction rates as function of substrate concentration (at fixed concentration of the remaining components of the reaction). The steady state kinetic data of the CPT I activity were determined by nonlinear regression analysis using the SigmaPlot ${ }^{\circledR} 10.0$ Technical Graphing Software plus the Enzyme Kinetics module 1.3. The Michaelis-Menten equation [36] was used to calculate kinetic parameters $\left(K_{\mathrm{m}}^{\mathrm{app}}\right.$ and $V_{\max }^{\mathrm{app}}$ ) of the enzyme reaction involving [U- $\left.{ }^{13} \mathrm{C}\right]-\mathrm{C} 16-\mathrm{CoA}$ and L-carnitine. The inhibition constant $K_{\mathrm{i}}^{\text {app }}$ was calculated by nonlinear regression of the respective inhibition curves, using the above mentioned software. 


\section{Results}

\subsection{CPT I activity in human fibroblasts}

The first aim of the present study was to elucidate whether valproyl-CoA had any effect on CPT I activity using control human skin fibroblasts permeabilized with digitonin, using a recently developed method [35]. The hepatic isoform of CPT I (CPT IA) is the only expressed in human fibroblasts [37,38]. The results of Fig. 1 show that valproyl-CoA is a potent inhibitor of CPT I comparable to malonyl-CoA, a well-known inhibitor of this enzyme. A similar result was also observed using $\Delta^{4}$-VP-CoA, resulting in decreased CPT I activity (data not shown).

When VP-CoA and $\Delta^{4}$-VP-CoA were tested as potential substrates for CPT I in fibroblasts, in the same experimental setup of Fig. 1, there was no formation of the respective carnitine esters (C8carnitine) (not shown).

Taken together, the results show that VP-CoA and $\Delta^{4}$-VP-CoA can inhibit the activity of CPT I.

\subsection{Malonyl-CoA sensitivity of the yeast expressed rCPT IA proteins}

In order to resolve whether valproyl-CoA inhibits CPT I via the same mechanism as malonyl-CoA, we decided to repeat the experiments of Fig. 1 using different recombinant isoforms of the rat CPT IA, characterised by different sensitivities towards malonyl-CoA. These proteins include the rCPT IA E3A isoform, which is virtually insensitive towards malonyl-CoA, and the S24A/Q30A isoform with an increased sensitivity towards malonyl-CoA when compared to wild-type rCPT IA. To this end, we expressed the three different isoforms in S. cerevisiae, and measured the activity of CPT IA in the homogenates in the presence of malonyl-CoA or valproyl-CoA. Rather than using wild type $S$. Cerevisiae, we expressed these three different CPT IA isoforms in the mutant strain of $S$. Cerevisiae in which the gene coding for carnitine acetyltransferase was disrupted.

In accordance with earlier data [2] the expressed rCPT IA proteins showed different sensitivities towards malonyl-CoA inhibition (Fig. 2-A). However, all these proteins were inhibited by VP-CoA 
in a similar extent, despite their different malonyl-CoA sensitivities (Fig. 2-B). The inhibition constants $\left(K_{\mathrm{i}}^{\mathrm{app}}\right)$ of VP-CoA for these proteins are characterized in Table 1.

The wild-type protein (rCPT IA WT) was inhibited by malonyl-CoA (Fig. 2-A), decreasing to 50\% of its initial activity with $0.1 \mathrm{mM}$ malonyl-CoA. A similar decrease in activity was obtained with VP-CoA as shown in Fig. 2-B.

The rCPT IA protein with increased sensitivity towards malonyl-CoA (S24A/Q30A) was inhibited at low malonyl-CoA concentrations, while VP-CoA gradually inhibited the activity of CPT I (Fig. $2 \mathrm{~A}$ and $\mathrm{B}$ ) as was found for the wild type protein.

Figure 2-A also shows that malonyl-CoA had virtually no effect on the activity of the mutant rCPT IA E3A which is insensitive to malonyl-CoA, as expected. In contrast to malonyl-CoA, however, VP-CoA did inhibit the activity of rCPT IA E3A, as shown in Fig. 2-B.

The results depicted in Fig. 2, demonstrate that VP-CoA inhibits the three rCPT IA isoforms in a different mechanism as malonyl-CoA, suggesting an interference on the palmitoyl-CoA binding site.

\subsection{Palmitoyl-CoA affinity of the yeast expressed rCPT IA proteins}

In order to test whether VP-CoA inhibits CPT IA by competing with palmitoyl-CoA (C16-CoA), we performed enzyme activity measurements using the three rCPT IA isoforms at different VPCoA concentrations.

The activity of all studied rCPT IA proteins decreased with increasing VP-CoA concentrations (Fig. 3), demonstrating the inhibition of this enzyme. Interestingly, VP-CoA was found to inhibit the activity of the malonyl-CoA insensitive rCPT IA E3A by a purely competitive mechanism, since the different lines of the Lineweaver-Burk plot (which correspond to different inhibitor concentrations) cross perfectly at one point of the y axes (Fig. 3-C). This result strongly supports our former hypothesis that VP-CoA interferes with the active site of the enzyme, since the regulatory domain is mutated in this protein. 
The kinetic parameters for the rCPT IA proteins were estimated and, as shown in Table 2 and from the Lineweaver-Burk plots inserted in Fig. 3, VP-CoA increased the $K_{\mathrm{m}}^{\mathrm{app}}$ for palmitoyl-CoA, suggesting a competitive inhibition mechanism of VP-CoA at the substrate binding on the rCPT IA.

In the case of rCPT IA WT protein, however, the lines of the Lineweaver-Burk plots did not cross the y axes at one specific point (Fig. 3-A). This prompted us to further study the effect VP-CoA on the malonyl-CoA sensitivity of these proteins. The results obtained with the rCPT IA proteins suggest that VP-CoA interferes with malonyl-CoA sensitivity by an unknown mechanism, but the main inhibition of the CPT IA by this VPA metabolite is at the C16-CoA binding site.

\subsection{Carnitine affinity of the yeast expressed rCPT IA proteins and interference with malonyl-CoA sensitivity}

In order to evaluate if VP-CoA has a similar inhibition mechanism of CPT IA as malonyl-CoA, we decided to further study its effect on the carnitine binding in the rCPT IA proteins, since it is known from literature that malonyl-CoA and carnitine bind closely in the CPT I protein and have inverse association $[20,39]$.

Figure 4 shows the results obtained in the carnitine affinity study of the three rCPT IA isoforms as function of both malonyl-CoA (panels A, C and E) and VP-CoA (panels B, D and F), respectively. The kinetic parameters for carnitine under these conditions were estimated by the MichaelisMenten equation (Table 3).

In the rCPT IA WT protein, the calculated $V_{\max }^{\text {app }}$ for carnitine decreased with malonyl-CoA, and was accompanied by an increase of almost 2.5 fold of the $K_{\mathrm{m}}^{\mathrm{app}}$ (Table 3 ), suggesting a mixed inhibition mechanism. In the malonyl-CoA insensitive rCPT IA E3A, however, the $V_{\max }^{\text {app }}$ was almost unchanged and the $K_{\mathrm{m}}^{\text {app }}$ for carnitine increased only 2 fold by malonyl-CoA, supporting what is known from literature that malonyl-CoA decreases the affinity for carnitine in the CPT IA [20,39]. As depicted in Fig. 4-E, the increase in the concentration of carnitine relieves the malonylCoA inhibition of CPT I in the mutant E3A, but not in the WT or the S24A/Q30A. 
On the other hand, for VP-CoA, a marked decrease of the $V_{\max }^{\text {app }}$ with no effect on the $K_{\mathrm{m}}^{\text {app }}$ for carnitine was observed in all rCPT IA recombinant proteins (Table 3). This result suggests that VP-CoA is a noncompetitive inhibitor of the rCPT IA for carnitine, likely interfering with this protein by a different mechanism as malonyl-CoA.

The interference of VP-CoA in the malonyl-CoA sensitivity was further evaluated in the rCPT IA proteins (Fig. 5). Indeed, VP-CoA was found to decreased the inhibition exerted by malonyl-CoA, resulting in an increase of the $K_{\mathrm{i}}^{\text {app }}$ value for malonyl-CoA with increasing VP-CoA concentration in the rCPT IA WT (Fig. 5-A) and S24A/Q30A (Fig. 5-B). The $K_{\mathrm{i}}^{\text {app }}$ of malonyl-CoA in the rCPT IA E3A couldn't obviously been calculated since this protein is insensitive to the inhibitor.

An increase of the $K_{\mathrm{i}}^{\text {app }}$ for malonyl-CoA induced by VP-CoA and $\Delta^{4}-\mathrm{VP}-\mathrm{CoA}$ was also observed in permeabilized fibroblasts as shown in Table 4, again suggesting that these CoA esters inhibit the CPT I activity but also interfere with the protein's sensitivity to malonyl-CoA by a still unknown mechanism.

Overall, the results presented show that malonyl-CoA and VP-CoA have different inhibition mechanisms for carnitine on the rCPT IA. Additionally, since VP-CoA was found to compete with palmitoyl-CoA, it is suggested that VP-CoA inhibits the CPT I protein at the catalytic domain. 
A significant weight gain and hepatotoxicity with steatosis have been frequently reported in patients treated with valproic acid (VPA) $[10,11,25,26]$. However, the mechanisms underlying these adverse VPA side effects are not completely understood. It has been well demonstrated that VPA interferes with several metabolic pathways, notably with mitochondrial energy metabolism. Previous studies from our group have shown a clear impairment of mitochondrial LC-FAO [11,28] induced by this drug, supported by an in vivo accumulation of specific acylcarnitines [40], which motivated the present work. Moreover, our recent findings showing the extramitochondrial activation of VPA to its CoA ester [9] sparked our interest on VP-CoA-CPT I interaction.

In the present report we show clear inhibition of CPT I activity by VP-CoA in control fibroblasts, which may partly account for the decreased rate of LC-FAO associated with the drug, and consequently to the fat accumulation in the liver. Moreover, we show that VP-CoA also interferes with the CPT I key regulator malonyl-CoA, mitigating the inhibition (as demonstrated by the increase of $K_{\mathrm{i}}^{\text {app }}$ for malonyl-CoA) and thus possibly affecting the energetic control of the cell. The activated form of the major microsomal metabolite of VPA, $\Delta^{4}$-VP-CoA, was also found to interfere with the CPT I activity in control fibroblasts, in a similar way as VP-CoA. The results obtained with these VPA metabolic intermediates stress the importance of considering not only the parent drug but also its metabolites as major triggering causes of the observed toxic effects.

To clarify the inhibitory mechanism of VP-CoA on the CPT I activity, three rCPT IA proteins with different sensitivities to malonyl-CoA were studied. The obtained results show that VP-CoA inhibited the malonyl-CoA insensitive rCPT IA protein (rCPT IA E3A), indicating an interference of VP-CoA at the catalytic domain of the protein. This hypothesis was further supported by the palmitoyl-CoA affinity study which showed that VP-CoA competes with this substrate for the wild type rCPT IA enzyme, as indicated by the respective increased $K_{\mathrm{m}}^{\mathrm{app}}$ and almost unchanged $V_{\max }^{\mathrm{app}}$ values. 
Malonyl-CoA was found to bind closely to the carnitine binding site in the CPT IA [20,37], displacing the binding of carnitine, which means that at higher carnitine concentrations, CPT IA is less sensitive to malonyl-CoA. We have shown that both malonyl-CoA and VP-CoA have different inhibition mechanisms for carnitine in the yeast expressed rCPT IA WT, respectively mixed and noncompetitive. Additionally, VP-CoA was not found to displace the binding of carnitine on the rCPT IA WT, as occurs with malonyl-CoA. Taken together, these results support the hypothesis that the interaction of VP-CoA with CPT IA is not analogous to the interaction of malonyl-CoA with CPT IA. Thus, VP-CoA most likely interacts with the acyl-CoA binding site of the protein.

In conclusion, the results described in this paper show that VP-CoA is an effective inhibitor of CPT IA by competing with palmitoyl-CoA for the same binding site. Interestingly, the CoA ester of VPA was not converted into the corresponding valproylcarnitine by CPT IA. Nevertheless, it is clear that VP-CoA has an effect on the CPT IA sensitivity for malonyl-CoA, but the underlying mechanism remains to be clarified. One hypothesis which requires further investigation is that the CoA ester may possibly induce a conformational change on the N-terminal region of CPT IA, resulting in changes on the $\mathrm{N}-\mathrm{C}$ intramolecular interactions, responsible for the malonyl-CoA sensitivity of the protein [2].

These findings may well be of great importance to explain the interference of VPA with FAO, especially since we have recently shown that VPA, which diffuses into mitochondria as free acid, is not only activated to VP-CoA inside mitochondria, but also in the extra-mitochondrial space [9]. The fate of cytosolic VP-CoA and $\Delta^{4}$-VP-CoA has not been clarified and it is an important object of study in our group. Since these CoA esters are not substrates of CPT IA, not forming carnitine esters, they can not be shuttled into mitochondria via the carnitine cycle and they may accumulate in the cytosol and can interfere with CPT IA, as shown in this study. In normal conditions, when glucose levels are low, hepatic malonyl-CoA concentration decreases and mitochondrial FAO becomes the energy source. However, in these conditions, if VP-CoA is present in the extramitochondrial compartment of the cell, it can inhibit the CPT IA activity, inducing lipid accumulation instead of oxidation. The microvesicular steatosis and the weight gain related with 
VPA therapy may be clinical signs potentially reflecting the imbalance in the energetic status of the cell.

\section{ACKNOWLEDGEMENTS}

This work was financially supported by Fundação para a Ciência e a Tecnologia (FCT), Lisboa, Portugal, by a grant awarded to Cátia C. P. Aires (SFRH/BD/22420/2005). 


\section{REFERENCES}

[1] Borthwick K, Jackson VN, Price NT, Zammit VA. The mitochondrial intermembrane loop region of rat carnitine palmitoyltransferase $1 \mathrm{~A}$ is a major determinant of its malonyl-CoA sensitivity. J Biol Chem 2006; 281: 32946-32952.

[2] Faye A, Borthwick K, Esnous C, Price NT, Gobin S, Jackson VN, et al. Demonstration of Nand C-terminal domain intramolecular interactions in rat liver carnitine palmitoyltransferase 1 that determine its degree of malonyl-CoA sensitivity. Biochem J 2005; 387: 67-76.

[3] McGarry JD, Leatherman GF, Foster DW. Carnitine palmitoyltransferase I: the site of inhibition of hepatic fatty acid oxidation by malonyl-CoA. J Biol Chem 1978; 253: 41284136.

[4] Cha SH, Hu Z, Chohnan S, Lane MD. Inhibition of hypothalamic fatty acid synthase triggers rapid activation of fatty acid oxidation in skeletal muscle. Proc Natl Acad Sci U.S.A. 2005; 102: $14557-14562$.

[5] Folmes CDL, Lopaschuk GD. Role of malonyl-CoA in heart disease and the hypothalamic control of obesity. Cardiovas Res 2007; 73: 278-287.

[6] Ruderman NB, Saha AK, Kraegen EW. Minireview: malonyl-CoA, AMP-activated protein kinase, and adiposity. Endocrinology 2003; 144: 5166-5171.

[7] Hu Z, Cha SH, Chohnan S, Lane MD. Hypothalamic malonyl-CoA as a mediator of feeding behaviour. Proc Natl Acad Sci U.S.A. 2003; 100: 12624-12629.

[8] Obici S, Feng Z, Arduini A, Conti R, Rossetti L. Inhibition of hypothalamic carnitine palmitoyltransferase-1 decreases food intake and glucose production. Nat Med 2003; 9: 756761.

[9] Aires CCP, Ruiter JPN, Luís PBM, ten Brink HJ, IJlst L, Tavares de Almeida I, et al. Studies on the extra-mitochondrial CoA-ester formation of valproic and $\Delta^{4}$-valproic acids. Biochim Biophys Acta 2007; 1771: 533-543. 
[10] Silva MFB, Ruiter JPN, Overmars H, Bootsma AH, van Gennip AH, Jakobs C, et al. Complete $\beta$-oxidation of valproate: cleavage of 3-oxovalproyl-CoA by a mitochondrial 3oxoacyl-CoA thiolase. Biochem J 2002; 362: 755-760.

[11] Silva MFB, Ruiter JPN, IJlst L, Jakobs C, Duran M, Tavares de Almeida I, et al. Differential effect of valproate and its $\Delta^{2}$ - and $\Delta^{4}$-unsaturated metabolites, on the $\beta$-oxidation rate of longchain and medium-chain fatty acids. Chem Biol Interact 2001; 137: 203-212.

[12] Britton CH, Mackey DW, Esser V, Foster DW, Burns DK, Yarnall DP, et al. Fine chromosome mapping of the genes for human liver and muscle carnitine palmitoyltransferase I (CPT IA and CPT IB). Genomics 1997; 40: 209-211.

[13] McGarry JD, Brown NF. The mitochondrial carnitine palmitoyltransferase system: from concept to molecular analysis. Eur J Biochem 1997; 244: 1-14.

[14] Price NT, van der Leij FR, Jackson VN, Corstorphine CG, Thomson R, Sorensen A, et al. A novel brain-expressed protein related to carnitine palmitoyltransferase I. Genomics 2002; 80: 433-442.

[15] Jackson VN, Price NT, Zammit VA. Specificity of the interactions between Glu-3, Ser-24, and Gln-30 within the N-terminal segment of rat liver mitochondrial overt carnitine palmitoyltransferase (L-CPT I) in determining the malonyl-CoA sensitivity of the enzyme. Biochemistry 2001; 40: 14629-14634.

[16] Price NT, Jackson VN, van der Leij FR, Cameron JM, Travers MT, Bartelds B, et al. Cloning and expression of the liver and muscle isoforms of ovine carnitine palmitoyltransferase 1 : residues within the $\mathrm{N}$-terminus of the muscle isoform influence the kinetic properties of the enzyme. Biochem J 2003; 372: 871-879.

[17] Pripp-Buus C, Cohen I, Kohl C, Esser V, McGarry JD, Girard J. Topological and functional analysis of the rat liver carnitine palmitoyltransferase 1 expressed in Saccharomyces cerevisiae. FEBS Lett 1998; 429: 173-178.

[18] Jackson VN, Zammit VA, Price NT. Identification of positive and negative determinants of malonyl-CoA sensitivity and carnitine affinity within the amino termini of rat liver- and muscle-type carnitine palmitoyltransferase I. J Biol Chem 2000; 275: 38410-38416. 
[19] Cohen I, Kohl C, McGarry JD, Girard J, Pripp-Buus C. The N-terminal domain of rat liver carnitine palmitoyltransferase 1 mediates import into the outer mitochondrial membrane and is essential for activity and malonyl-CoA sensitivity. J Biol Chem 1998; 273: 29896-29904.

[20] McGarry JD, Mills SE, Long CS, Foster DW. Observations on the affinity for carnitine, and malonyl-CoA sensitivity, of carnitine palmitoyltransferase I in animal and human tissues. Biochem J 1983; 214: 21-28.

[21] Shi J, Zhu H, Arvidson DN, Cregg JM, Woldegiorgis G. Deletion of the conserved first 18 N-terminal amino acid residues in rat liver carnitine palmitoyltransferase I abolishes malonyl-CoA sensitivity and binding. Biochemistry 1998; 37: 11033-11038.

[22] Morillas M, Gomez-Puertas P, Bentebibel A, Selles E, Casals N, Valencia A, et al. Identification of conserved amino acid residues in rat liver carnitine palmitoyltransferase I critical for malonyl-CoA inhibition. J Biol Chem 2003; 278: 9058-9063.

[23]Faye A, Esnous C, Price NT, Onfray MA, Girard J, Prip-Buus C. Rat liver carnitine palmitoyltransferase 1 forms an oligomeric complex within the outer mitochondrial membrane. J Biol Chem 2007; 282: 26908-26916.

[24]Zammit VA. Carnitine palmitoyltransferase 1: central to cell function. IUBMB Life 2008; 60: $347-354$

[25] Luef GJ, Lechleitner M, Bauer G, Trinka E, Hengster P. Valproic acid modulates islet cell insulin secretion: a possible mechanism of weight gain in epilepsy patients. Epilepsy Res 2003; 55: 53-58.

[26] Pylvanen V, Pakarinen A, Knip M, Isojarvi J. Insulin-related metabolic changes during treatment with valproate in patients with epilepsy. Epilepsy Behav 2006; 8: 643-648.

[27] Verrotti A, la Torre R, Trotta D, Mohn A, Chiarelli F. Valproate-induced insulin resistance and obesity in children. Horm Res 2009; 71: 125-131.

[28] Silva MFB, Jakobs C, Duran M, Tavares de Almeida I, Wanders RJA. Valproate induces in vitro accumulation of long-chain fatty acylcarnitines. Mol Genet Metab 2001; 73: 358-361. 
[29] Rasmussen JT, Borchers T, Knudsen J. Comparison of the binding affinities of acyl-CoAbinding protein and fatty-acid-binding protein for long-chain acyl-CoA esters. Biochem $\mathbf{J}$ 1990; 265: 849-855.

[30] Silva MFB, Ruiter JPN, Ijlst L, Allers P, ten Brink H, Jakobs C, et al. Synthesis and intramitochondrial levels of valproyl-coenzyme A metabolites. Anal Biochem 2001; 290: 6067.

[31] Rettenmeyer AW, Prickett KS, Gordon WP, Bjorge SM, Chang S-L, Levy RH, et al. Studies on the biotransformation in the perfused rat liver of 2-n-propyl-4-pentenoic acid, a metabolite of the antiepileptic drug valproic acid: Evidence for the formation of chemically reactive intermediates. Drug Metab Dispos 1985; 13: 81-96.

[32] Wanders RJ, Ruiter JP, Wijburg FA. Studies on mitochondrial oxidative phosphorylation in permeabilized human skin fibroblasts: application to mitochondrial encephalomyopathies. Biochim Biophys Acta 1993; 1181: 219-222.

[33] Smith PK, Krohn RI, Hermanson GT, Mallia AK, Gartner FH, Provenzano MD, et al. Measurement of protein using Bicinchoninic acid. Anal Biochem 1985; 150: 76-85.

[34] Spaan AN, IJlst L, van Roermund CWT, Wijburg FA, Wanders RJA, Waterham HR. Identification of the human mitochondrial FAD transporter and its potential role in multiple acyl-CoA dehydrogenase deficiency. Mol Genet Metab 2005; 86: 441-447.

[35] van Vlies N, Ruiter JP, Doolaard M, Wanders RJA, Vaz FM. An improved enzyme assay for carnitine palmitoyl transferase I in fibroblasts using tandem mass spectrometry. Mol Genet Metab 2007; 90: 24-29.

[36] Brooks SPJ. Enzymes - The Basis of Catalysis in Functional Metabolism: Regulation and Adaptation. In: Storey KB editor. John Wiley \& Sons, Inc., New Jersey, 2004.

[37] Britton CH, Schultz RA, Zhang B, Esser V, Foster DW, McGarry JD. Human liver mitochondrial carnitine palmitoyltransferase I: characterization of its cDNA and chromosomal localization and partial analysis of the gene. Proc Natl Acad Sci USA 1995; 92: 1984-1988. 
[38] IJlst L, Mandel H, Oostheim W, Ruiter JPN, Gutman A, Wanders RJA. Molecular basis of hepatic carnitine palmitoyltransferase I deficiency. J Clin Invest 1998; 102: 527-531.

[39]Lopez-Viñas E, Bentebibel A, Gurunathan C, Morillas M, Arriaga D, Serra D, et al. Definition by functional and structural analysis of two malonyl-CoA sites in carnitine palmitoyltransferase 1A. J Biol Chem 2007; 282: 18212-18224.

[40] Silva MFB, Selhorst J, Overmars H, van Gennip AH, Maya M, Wanders RJA, et al. Characterization of plasma acylcarnitines in patients under valproate monotherapy using ESIMS/MS. Clin Biochem 2001; 34: 635-638. 
Table 1: Estimated inhibition constant $\left(K_{\mathrm{i}}^{\text {app }}\right)$ values of valproyl-CoA for the rat CPT IA wildtype (WT), mutants with increased sensitivity (S24A/Q30A) and insensitive (E3A) to malonylCoA. Yeast transformed with rCPT IA were used to evaluate this enzyme activity at different valproyl-CoA concentration $(0-100 \mu \mathrm{M})$ with $25 \mu \mathrm{M}\left[\mathrm{U}_{-}{ }^{13} \mathrm{C}\right]$-palmitoyl-CoA, $0.5 \mathrm{mM} \mathrm{L}-$ carnitine and without malonyl-CoA. (Results are the mean +/- SD of duplicates from two independent experiments).

\begin{tabular}{cc}
\hline rCPT IA & $\boldsymbol{K}_{\mathbf{i}}^{\text {app }}$ Valproyl-CoA $(\boldsymbol{\mu M})$ \\
\hline WT & $57 \pm 7$ \\
S24A/Q30A & $70 \pm 28$ \\
E3A & $51 \pm 25$ \\
\hline
\end{tabular}




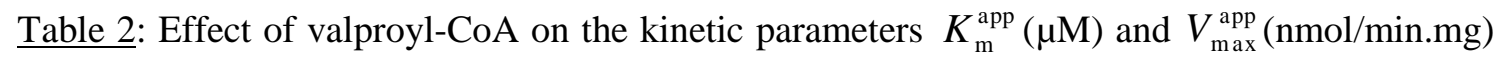
of the rat CPT IA wild-type (WT), the sensitive rCPT IA to malonyl-CoA (S24A/Q30A) and the insensitive to malonyl-CoA (E3A) for palmitoyl-CoA as substrate. (Results are the mean +/- SD of duplicates from two independent experiments; nd - not determined since the estimated $K_{\mathrm{m}}$ value is higher than $700 \mu \mathrm{M})$.

\begin{tabular}{|c|c|c|c|c|c|c|}
\hline & \multicolumn{6}{|c|}{ Palmitoyl-CoA } \\
\hline & \multicolumn{2}{|c|}{ rCPT IA WT } & \multicolumn{2}{|c|}{ rCPT IA S24A/Q30A } & \multicolumn{2}{|c|}{ rCPT IA E3A } \\
\hline & $K_{\mathrm{m}}^{\mathrm{app}}$ & $V_{\max }^{\text {app }}$ & $K_{\mathrm{m}}^{\mathrm{app}}$ & $V_{\max }^{\text {app }}$ & $K_{\mathrm{m}}^{\mathrm{app}}$ & $V_{\max }^{\text {app }}$ \\
\hline Control & $48 \pm 11$ & $61 \pm 13$ & $66 \pm 9$ & $77 \pm 10$ & $52 \pm 5$ & $79 \pm 1$ \\
\hline \multicolumn{7}{|l|}{ Valproyl-CoA $(\boldsymbol{\mu M})$} \\
\hline 10 & $51 \pm 33$ & $44 \pm 9$ & $54 \pm 6$ & $52 \pm 16$ & $105 \pm 19$ & $113 \pm 24$ \\
\hline 30 & $219 \pm 107$ & $127 \pm 66$ & $216 \pm 92$ & $138 \pm 54$ & $187 \pm 122$ & $136 \pm 75$ \\
\hline 100 & $289 \pm 50$ & $115 \pm 12$ & nd & nd & $209 \pm 75$ & $117 \pm 35$ \\
\hline
\end{tabular}




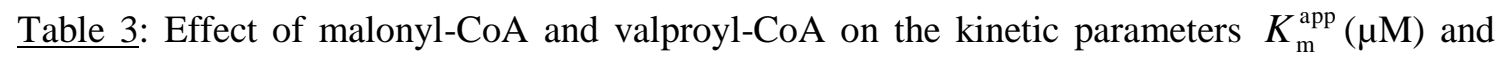
$V_{\max }^{\text {app }}(\mathrm{nmol} / \mathrm{min} . \mathrm{mg}$ ) of the rat CPT IA wild-type (WT), the sensitive rCPT IA to malonyl-CoA (S24A/Q30A) and the insensitive to malonyl-CoA (E3A) for carnitine as a substrate. (The experiment was performed in duplicate and the results are the mean $+/-\mathrm{SD}$ ).

\begin{tabular}{lccccc}
\hline \multicolumn{4}{c}{ L-Carnitine } \\
\hline \multicolumn{2}{c}{ rCPT IA WT } & \multicolumn{2}{c}{ rCPT IA S24A/Q30A } & rCPT IA E3A \\
$K_{\mathrm{m}}^{\text {app }}$ & $V_{\text {max }}^{\text {app }}$ & $K_{\mathrm{m}}^{\text {app }}$ & $V_{\text {max }}^{\text {app }}$ & $K_{\mathrm{m}}^{\text {app }}$ & $V_{\text {max }}^{\text {app }}$
\end{tabular}

$\begin{array}{lllllll}\text { Control } & 38.5 \pm 14.8 & 18.8 \pm 2.4 & 27.5 \pm 0.5 & 19.3 \pm 1.4 & 24.4 \pm 6.1 & 22.9 \pm 5.5\end{array}$

\begin{tabular}{|c|c|c|c|c|c|c|}
\hline \multicolumn{7}{|l|}{ Malonyl-CoA $(\mu \mathrm{M})$} \\
\hline 15 & $34.7 \pm 8.0$ & $16.4 \pm 0.9$ & $40.8 \pm 6.6$ & $12.3 \pm 0.5$ & $31.5 \pm 6.5$ & $24.5 \pm 1.1$ \\
\hline 50 & $68.8 \pm 7.8$ & $15.1 \pm 0.5$ & $62.2 \pm 8.2$ & $7.6 \pm 0.3$ & $33.5 \pm 3.2$ & $25.7 \pm 0.5$ \\
\hline 150 & $64.9 \pm 11.9$ & $10.8 \pm 0.5$ & $79.5 \pm 14.2$ & $4.3 \pm 0.2$ & $38.5 \pm 4.1$ & $23.6 \pm 0.6$ \\
\hline 500 & $93.8 \pm 24.9$ & $4.9 \pm 0.4$ & $83.3 \pm 6.2$ & $2.0 \pm 0.0$ & $65.1 \pm 6.2$ & $23.5 \pm 0.6$ \\
\hline \multicolumn{7}{|l|}{ Valproyl-CoA $(\mu \mathrm{M})$} \\
\hline 10 & $31.0 \pm 4.1$ & $14.6 \pm 0.4$ & $29.3 \pm 4.2$ & $16.0 \pm 0.5$ & $26.8 \pm 5.8$ & $17.4 \pm 0.8$ \\
\hline 30 & $31.9 \pm 9.8$ & $12.1 \pm 0.8$ & $12.9 \pm 4.4$ & $11.7 \pm 0.6$ & $20.6 \pm 4.1$ & $12.5 \pm 0.5$ \\
\hline 100 & $39.4 \pm 4.9$ & $8.9 \pm 0.3$ & $16.6 \pm 5.5$ & $8.6 \pm 0.5$ & $26.5 \pm 4.5$ & $9.5 \pm 0.3$ \\
\hline
\end{tabular}


Table 4: Estimated inhibition constant $\left(K_{\mathrm{i}}^{\text {app }}\right)$ values of malonyl-CoA for the CPT I in human control fibroblasts with increasing concentrations of valproyl-CoA $(10-100 \mu \mathrm{M})$ and $\Delta^{4}$-valproyl$\operatorname{CoA}(100-250 \mu \mathrm{M})$.

$K_{\mathrm{i}}^{\text {app }}$ Malonyl-CoA (mM)

$\begin{array}{ll}\text { Controls } & 0.029-0.078\end{array}$

\begin{tabular}{cc}
\hline Valproyl-CoA $(\boldsymbol{\mu M})$ & \\
\hline $\mathbf{1 0}$ & 0.095 \\
$\mathbf{5 0}$ & 0.121 \\
$\mathbf{1 0 0}$ & 0.273 \\
\hline$\Delta^{4}$-Valproyl-CoA $(\boldsymbol{\mu M})$ & \\
\hline $\mathbf{1 0 0}$ & 0.188 \\
$\mathbf{2 5 0}$ & 0.138 \\
\hline
\end{tabular}


Fig. 1: Sensitivity of the CPT IA activity in human control fibroblasts for (A) malonyl-CoA and (B) valproyl-CoA. The CPT I activity was measured in digitonin permeabilized control fibroblasts after incubation for 10 minutes at $37^{\circ} \mathrm{C}$ with $25 \mu \mathrm{M}\left[\mathrm{U}-{ }^{13} \mathrm{C}\right]$-palmitoyl-CoA, $0.5 \mathrm{mM}$ L-carnitine, $5 \mathrm{mM} \mathrm{KCN}$ and increasing concentrations of malonyl-CoA $(0-1 \mathrm{mM})$, and VP$\operatorname{CoA}(0-100 \mu \mathrm{M})$. 

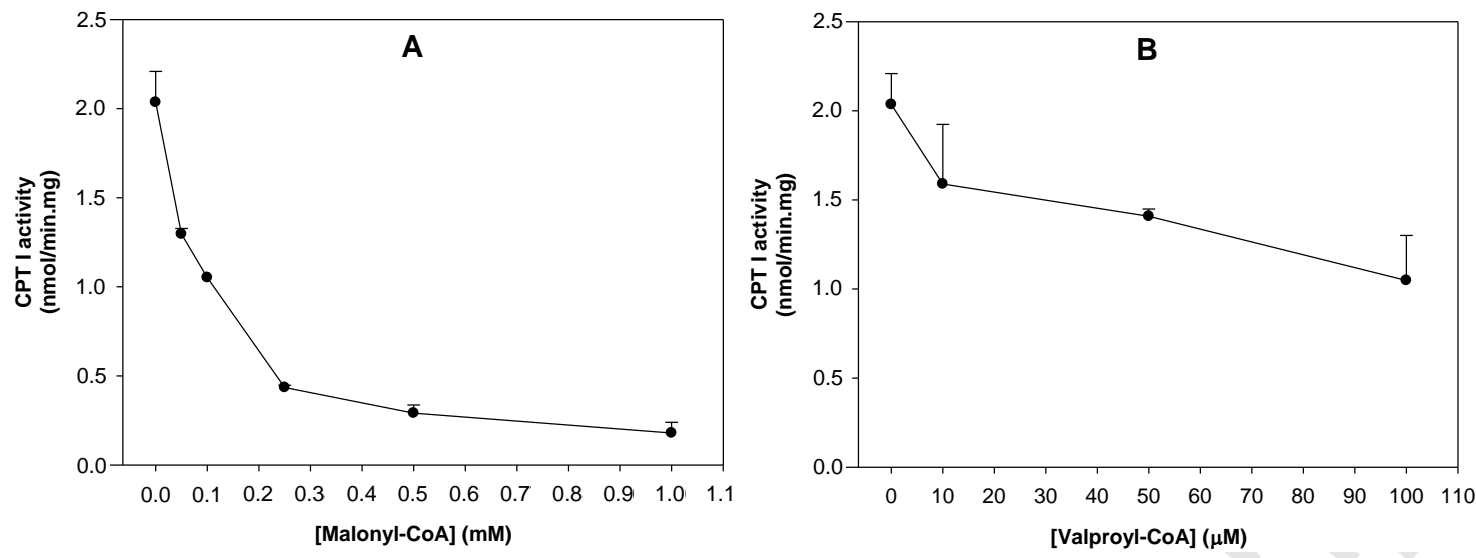
Fig. 2: Effect of (A) malonyl-CoA and (B) valproyl-CoA on the activity of three yeast expressed rat CPT IA isoforms (rCPT IA WT, rCPT IA S24A/Q30A with increased sensitivity to malonyl-CoA, and rCPT IA E3A insensitive to malonyl-CoA). The respective activity was measured after incubation with $25 \mu \mathrm{M}\left[\mathrm{U}_{-}{ }^{13} \mathrm{C}\right]-\mathrm{C} 16-\mathrm{CoA}$ and different concentrations of (A) malonyl-CoA $(0-0.5 \mathrm{mM})$ and $(\mathbf{B})$ valproyl-CoA $(0-100 \mu \mathrm{M})$ respectively. (Results are the mean $+/$ - SD of duplicates from two independent experiments). 

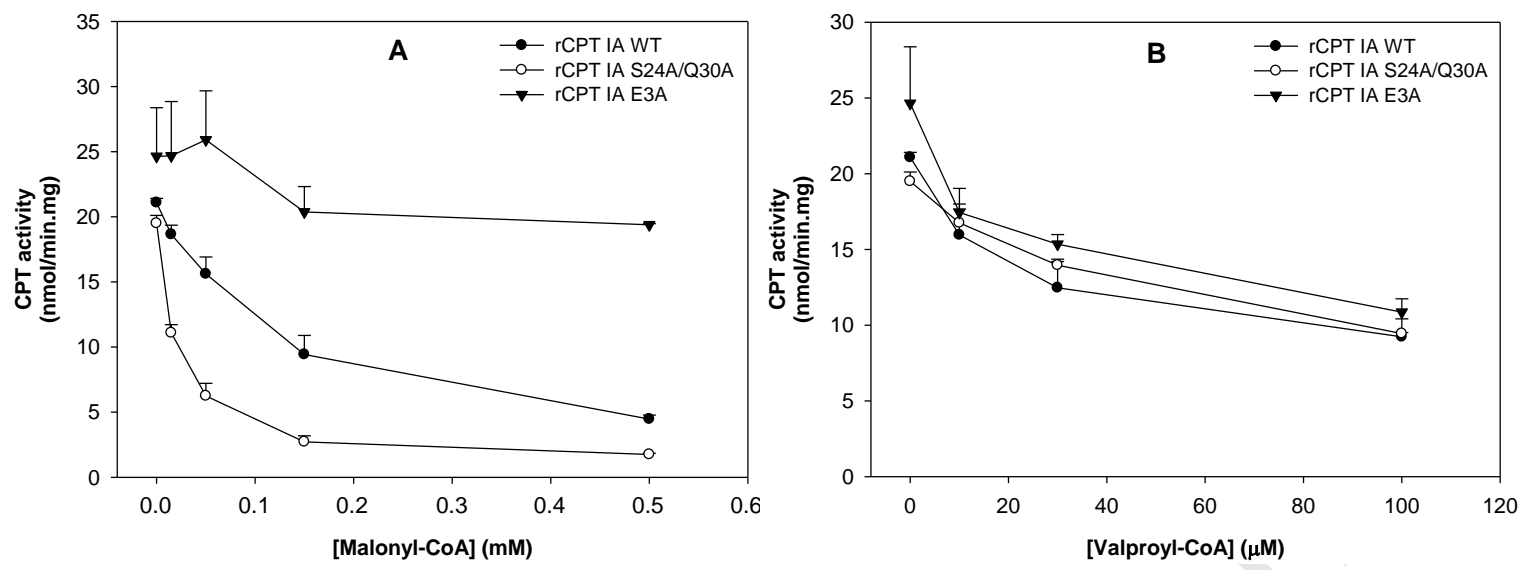
Fig. 3: Effect of valproyl-CoA (VP-CoA) on the affinity of the yeast expressed rat CPT IA proteins for palmitoyl-CoA (C16-CoA). The affinity for C16-CoA $(0-50 \mu \mathrm{M})$ of the (A) rCPT IA WT, (B) rCPT IA S24A/Q30A, and (C) rCPT IA E3A was measured in the presence of different VP-CoA concentrations $(\bullet 0 \mu \mathrm{M}, \circ 10 \mu \mathrm{M}, \boldsymbol{\nabla} 30 \mu \mathrm{M}$ and $\triangle 100 \mu \mathrm{M})$. The Lineweaver-Burk plots (inserts) for the three isoforms of rCPT IA were constructed by linearization of the correspondent substrate affinity curves. (Results are the mean +/- SD of duplicates from two independent experiments). 

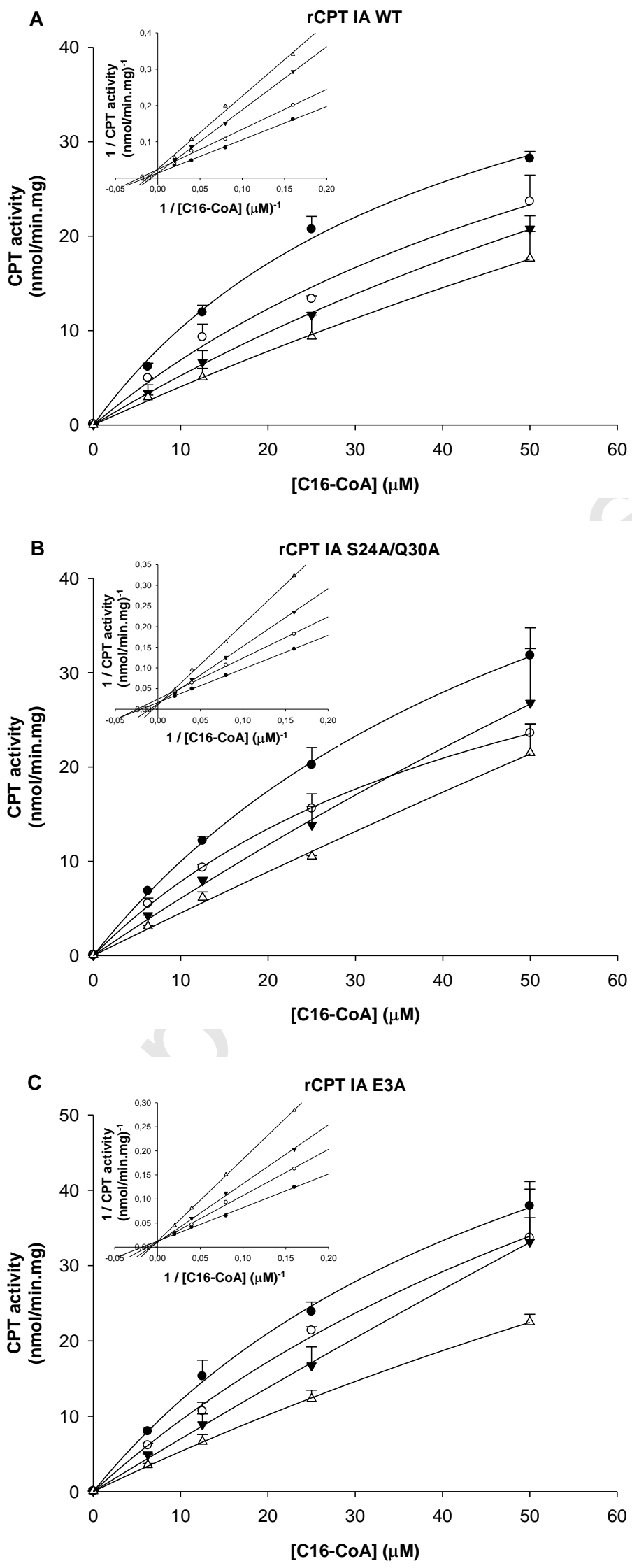
Fig. 4: Effect of malonyl-CoA (Mal-CoA) and valproyl-CoA (VP-CoA) on the affinity for carnitine of the yeast expressed rat CPT IA proteins. The affinity for carnitine $(0-750 \mu \mathrm{M})$ of the rCPT IA WT (A, B), rCPT IA S24A/Q30A (C, D) and rCPT IA E3A (E, F) was studied in the presence of Mal-CoA $(0-500 \mu \mathrm{M})$ (left panels) and VP-CoA $(0-100 \mu \mathrm{M})$ (right panels). 

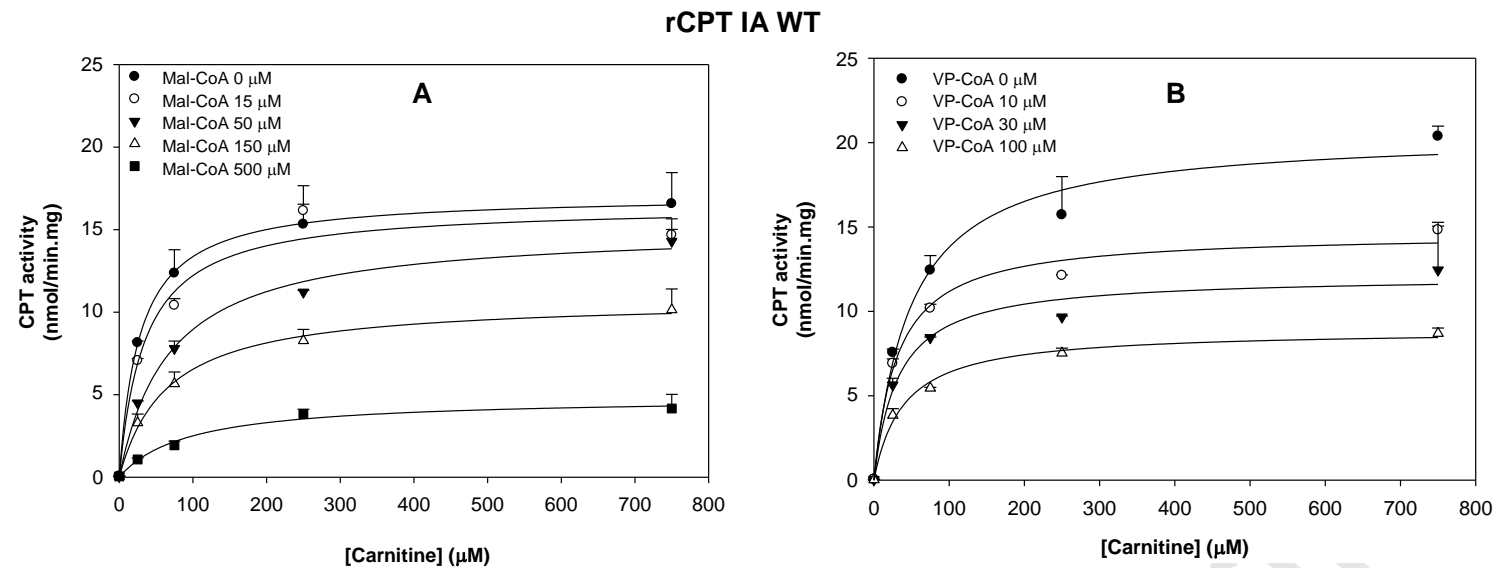

rCPT IA S24A/Q30A
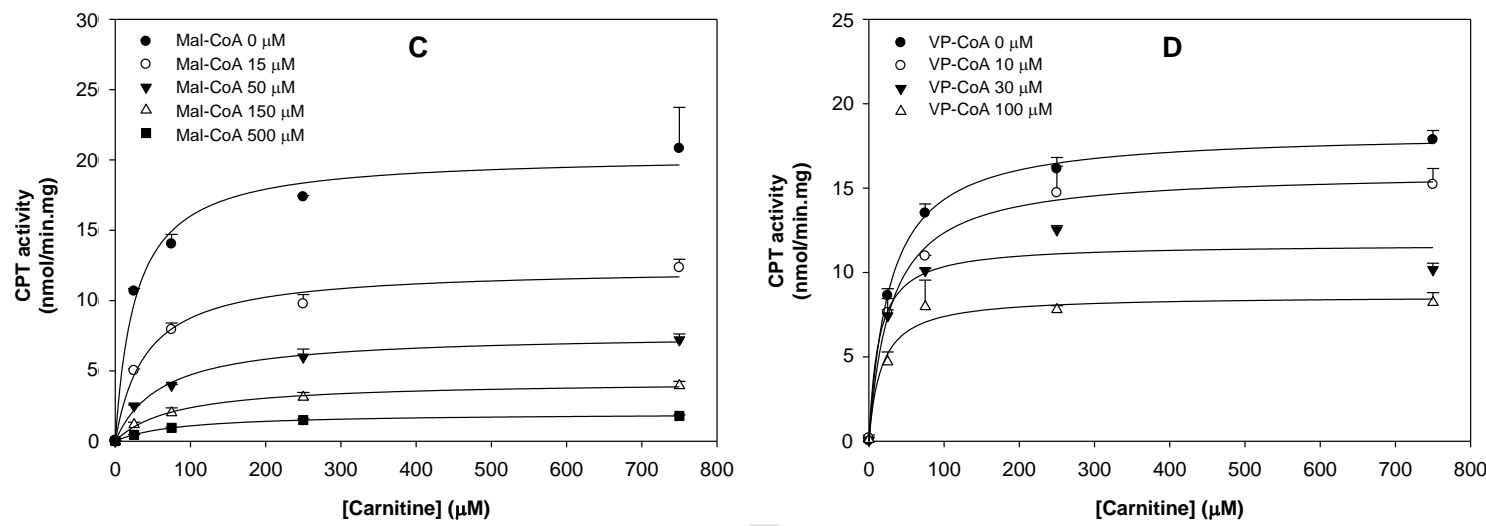

rCPT IA E3A

Fig. 5: Estimated $K_{\mathrm{i}}^{\text {app }}$ values of malonyl-CoA for the rat CPT IA proteins (A) wild-type (WT) and (B) with increased sensitivity for malonyl-CoA (S24A/Q30A) in the presence of valproylCoA $(0-100 \mu \mathrm{M})$. (Results are the mean $+/-$ SD of duplicates from two independent experiments) 

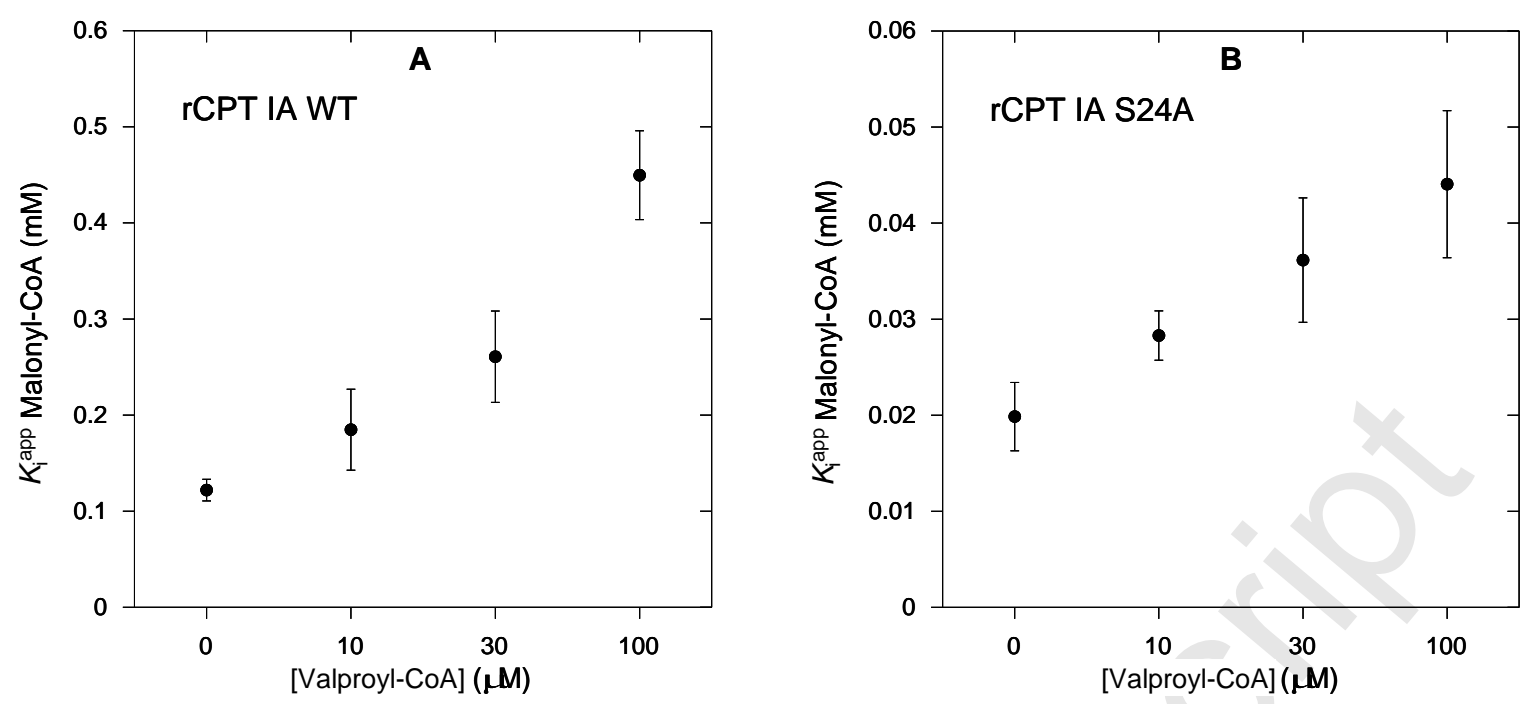

Page 34 of 35 


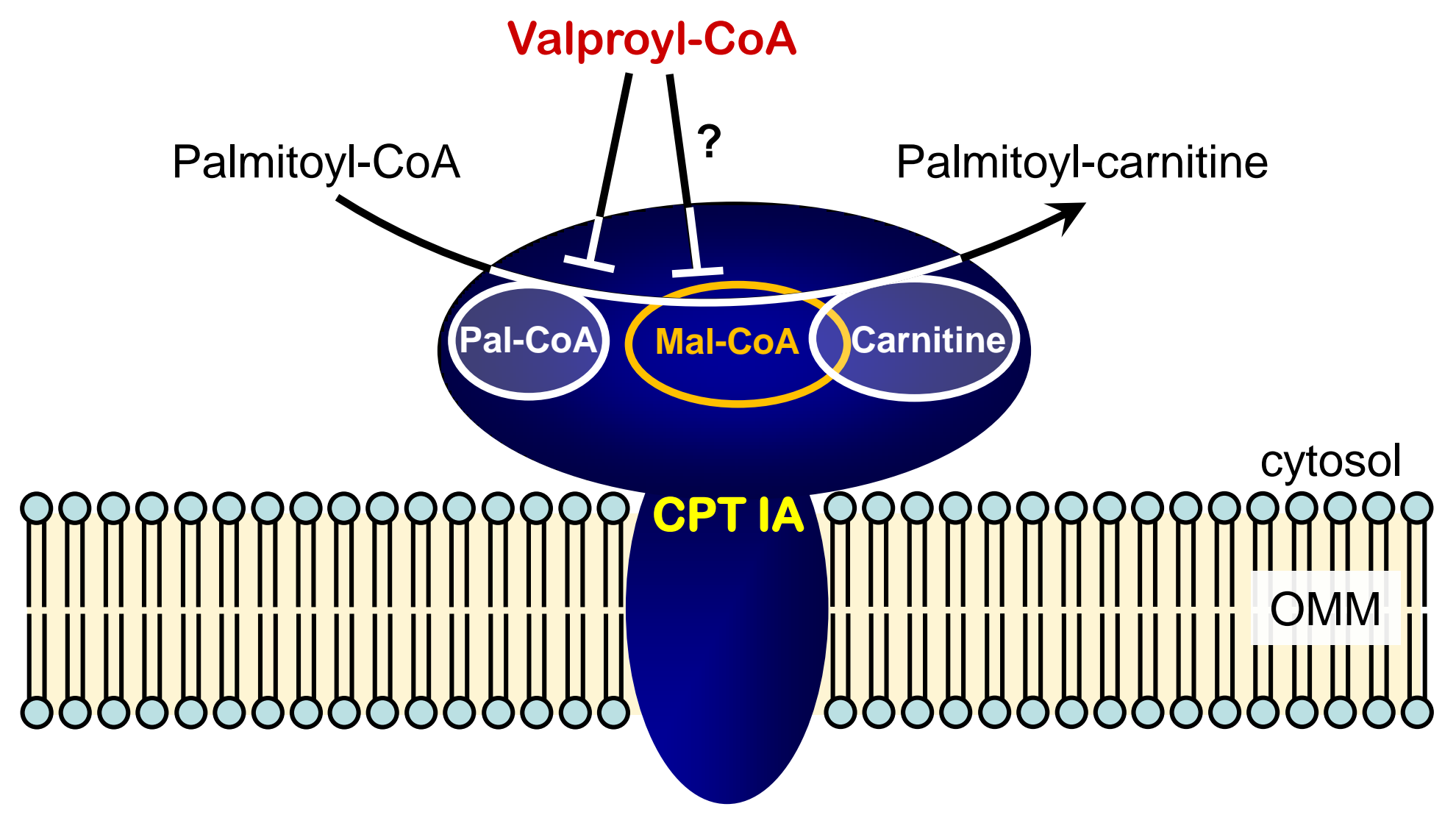

Proposed mechanism of CPT IA inhibition by Valproyl-CoA. Valproyl-CoA competitively inhibits palmitoyl-CoA binding and affects the physiological inhibition of malonyl-CoA. 